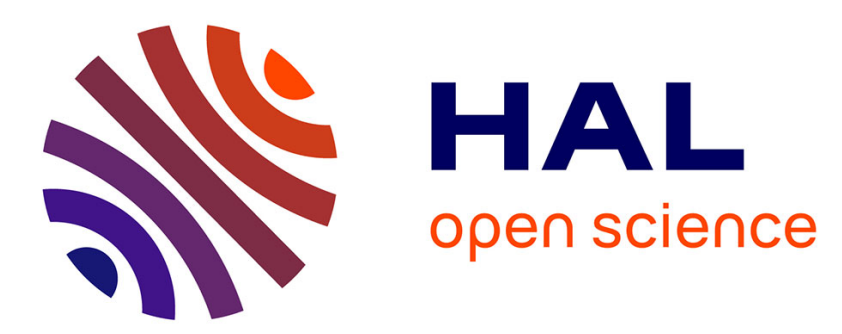

\title{
Mesures et modélisations des déformations élastiques autour de nanoprécipités
}

M. Le Fournier, Joël Douin, Christophe Gatel, Florence Pettinari-Sturmel, P. Donnadieu

\section{- To cite this version:}

M. Le Fournier, Joël Douin, Christophe Gatel, Florence Pettinari-Sturmel, P. Donnadieu. Mesures et modélisations des déformations élastiques autour de nanoprécipités. Revue de Métallurgie, 2012, 109 (6), pp.409-414. 10.1051/metal/2012004 . hal-02062137

\section{HAL Id: hal-02062137 https://hal.science/hal-02062137}

Submitted on 21 Jun 2019

HAL is a multi-disciplinary open access archive for the deposit and dissemination of scientific research documents, whether they are published or not. The documents may come from teaching and research institutions in France or abroad, or from public or private research centers.
L'archive ouverte pluridisciplinaire HAL, est destinée au dépôt et à la diffusion de documents scientifiques de niveau recherche, publiés ou non, émanant des établissements d'enseignement et de recherche français ou étrangers, des laboratoires publics ou privés. 


\title{
Mesures et modélisations des déformations élastiques autour de nanoprécipités
}

\author{
M. Le Fournier ${ }^{1,2}$, J. Douin ${ }^{1}$, C. Gatel ${ }^{1,2}$, F. Pettinari-Sturmel ${ }^{1,2}$ \\ et P. Donnadieu ${ }^{3}$ \\ 1 CEMES-CNRS, 29 rue Jeanne Marvig, 31055 Toulouse Cedex 04, France \\ e-mail : marion.lefournier@cemes.fr \\ 2 Université de Toulouse, UPS, 29 rue Jeanne Marvig, 31055 Toulouse Cedex 04, France \\ 3 SIMAP, INP Grenoble-CNRS-UJF, BP 75, 39402 Saint Martin d'Hères Cedex, France
}

Mots-clés :

Microscopie electronique haute résolution; nanoparticules ; modélisation de champ de déformation; dislocation ; alliage d'Aluminium

Key words:

High-resolution electron microscopy; nanoparticles; strain field modeling; dislocation; Aluminum alloys

Reçu le 27 octobre 2011 Accepté le 24 janvier 2012

\begin{abstract}
Résumé - La mesure précise des champs de contraintes autour de précipités inclus dans une matrice cristalline est nécessaire à la compréhension de l'interaction entre les dislocations et les précipités de matériaux structurellement durcis. Ces interactions contrôlent le mouvement des dislocations, et donnent donc des informations sur les propriétés mécaniques des matériaux. Les champs de contraintes autour des précipités de petite taille peuvent être obtenus à partir d'images de microscopie électronique en transmission en haute résolution (METHR) en utilisant la méthode des phases géométrique (GPA). Cette méthode est utilisable de façon générale pour caractériser les contraintes à l'échelle nanométrique autour des précipités de structure complexe ou inconnue et est appliquée ici dans les cas de l'alliage d'aluminium 2198. Dans ce matériau, il est montré que les champs de déformation autour d'un nanoprécipité en forme de disque et d'épaisseur faible peuvent être modélisés par ceux d'une dislocation dissociée.
\end{abstract}

\begin{abstract}
Measurement and modeling of the elastic strain around nanoprecipitates. Precise measurements of the strain fields around small precipitates embedded in a crystalline matrix is essential to understand the interaction of dislocations with precipitates of structurally hardened materials. This interaction controls the dislocation motion through an array of precipitates that are responsible for structural hardening of the material. The displacement and strain fields around nanosized particles can be obtained from high-resolution electron micrographs using the geometric phase analysis (GPA). The method is generally applicable to the characterization of the strain in nanostructured materials, including those with complex or unknown structures and is emphasized here in the case of the 2198 Aluminum alloy. In this material, it is shown that the strain field around a disc-shaped nanoprecipitate can be modeled to some extend by a dissociated dislocation.
\end{abstract}

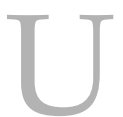
n des enjeux actuels du secteur aéronautique est d'augmenter les propriétés mécaniques spécifiques des matériaux de structure [1]. Les alliages d'aluminium $\mathrm{Al}-\mathrm{Li}-\mathrm{Cu}$ sont connus pour leur faible densité et leurs bonnes propriétés mécaniques dues à la présence de lithium et à l'application d'une déformation plastique avant le traitement thermique qui permet la nucléation de précipités de structure hexagonale de type $\mathrm{T}_{1}[2,3]$ (Fig. 1). Les propriétés mécaniques macroscopiques de ces alliages sont régies par leur microstructure. En effet, il est bien connu que les précipités s'opposent au déplacement des dis- locations, qui portent la déformation, permettant une augmentation de la résistance mécanique du matériau. La mesure précise des champs de contraintes autour de précipités inclus dans une matrice cristalline apparaît donc nécessaire à la compréhension de l'interaction entre les dislocations et les précipités de matériaux structurellement durcis. Les champs de déformation autour des précipités peuvent être obtenus à partir d'images de microscopie électronique en transmission en haute résolution (METHR) en utilisant la méthode des phases géométriques (GPA) [4]. Dans l'alliage d'aluminium 6056, les champs de déformation créés 


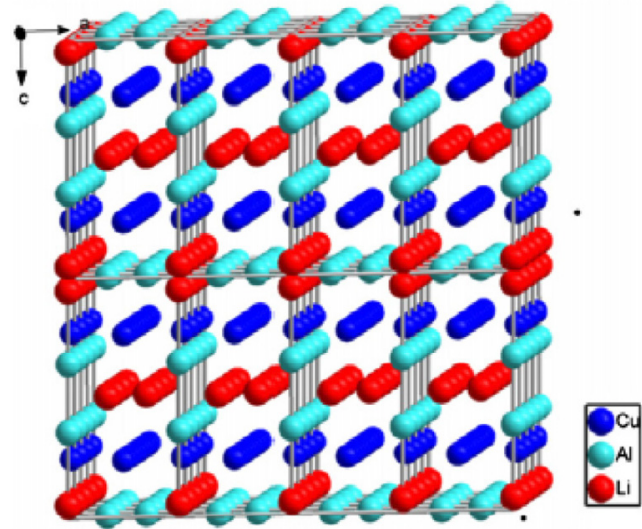

Fig. 1. Illustration de la structure $T_{1}$ vue dans le plan a-c proposée par Donnadieu [2].

Fig. 1. Illustration of the $T_{1}$ structure as seen in the a-c plane proposed by Donnadieu [2].

par un précipité en forme d'aiguille et de section transverse rectangulaire de taille nanométrique, ont été modélisés par ceux dus à un dipôle de dislocations coin [5]. Le but de ce papier est de montrer qu'il est possible, d'une part d'utiliser GPA pour mesurer les déformations imposées à la matrice d'aluminium par des précipités en forme de disques, et $\mathrm{d}$ 'autre part de montrer que les déformations autour de ces précipités sont, au moins pour le bord du précipité, proches de celles générées par une dislocation.

\section{Techniques}

L'alliage d'aluminium 2198-T8 contient $3 \% \mathrm{~m}$. Cu, $1 \% \mathrm{~m}$. Li et de petites quantités $\mathrm{d}^{\prime} \mathrm{Ag}, \mathrm{Mg}$ et $\mathrm{Zr}$. Après une mise en solution et une trempe à l'eau, cet alliage a subi une déformation plastique de $2 \%$ et un traitement thermique à $155^{\circ} \mathrm{C}$ pendant $16 \mathrm{~h}$ : ce traitement permet de former des précipités identifiés comme la phase $\mathrm{T}_{1}-\mathrm{Al}_{2} \mathrm{CuLi}$. Ces précipités se présentent sous la forme de disques ou lentilles qui précipitent parallèlement aux plans $\{111\}$ de la matrice d'aluminium. Leur épaisseur est d'environ $1 \mathrm{~nm}$ et leurs diamètres varient de 20 à $100 \mathrm{~nm}$. Les lames minces de microscopie sont obtenues par polissage mécanique puis électrolytique. Les colonnes atomiques de la matrice proches des précipités sont imagées en METHR. Le microscope utilisé est un microscope FEI Tecnai F20 (SACTEM-Toulouse) fonctionnant à $200 \mathrm{kV}$, équipé d'un canon à émission de champ (FEG) et d'un correcteur $\mathrm{d}$ 'aberration sphérique. La résolution point à point de ce microscope est de 0,12 nm.

Les champs de déplacement de la matrice d'aluminium peuvent être obtenus en utilisant la technique d'analyse des phases géométriques GPA à partir des images en haute résolution [4]. GPA combine des informations obtenues dans l'espace réel et l'espace de Fourier : une image de METHR est décomposée en une somme d'images de franges, chaque système de franges étant associée à un vecteur d'onde $g$ du réseau réciproque. Lorsque le cristal est déformé, la distance entre les franges change par rapport au cristal non déformé, ce qui se traduit par un déphasage de la phase de cette onde $g$ comparé à la phase de cette même onde dans un cristal non déformé. Le déphasage $P_{g}$ et le champ de déplacement $u$ sont reliés par : $P_{g}=-2 \pi \vec{g} \bullet \vec{u}[4]$. La projection du champ de déplacement dans la direction de l'onde $g$ est obtenue en chaque point de l'image, et en sélectionnant deux vecteurs d'ondes différents, le champ de déplacement dans le plan perpendiculaire au faisceau d'électrons est mesuré. Le champ de déformation est alors obtenu par dérivation du champ de déplacement :

$$
\varepsilon_{i j}=\frac{1}{2}\left(\frac{\partial u_{i}}{\partial x_{j}}+\frac{\partial u_{j}}{\partial x_{i}}\right)
$$

Dans notre cas, les déformations mesurées autour des précipités sont petites et le rapport signal sur bruit est généralement faible. Nous avons cependant choisi d'utiliser GPA avec une résolution spatiale assez grande $(\leq 2 \mathrm{~nm})$ pour lisser les fluctuations liées aux conditions d'observation Pour une résolution spatiale de $2 \mathrm{~nm}, \mathrm{l}^{\prime}$ erreur, définie comme la déviation standard de la déformation mesurée sur une aire de $5 \times 5 \mathrm{~nm}^{2}$, varie de $0,1 \%$ à $0,2 \%[5,6]$.

\section{Résultats}

La figure 2 montre un exemple représentatif de deux précipités dans l'alliage d'aluminium 2198-T8 observé dans l'axe de zone [110] Dans cet alliage, les précipités sont nombreux et modifient de manière caractéristique la matrice. La présence d'un 


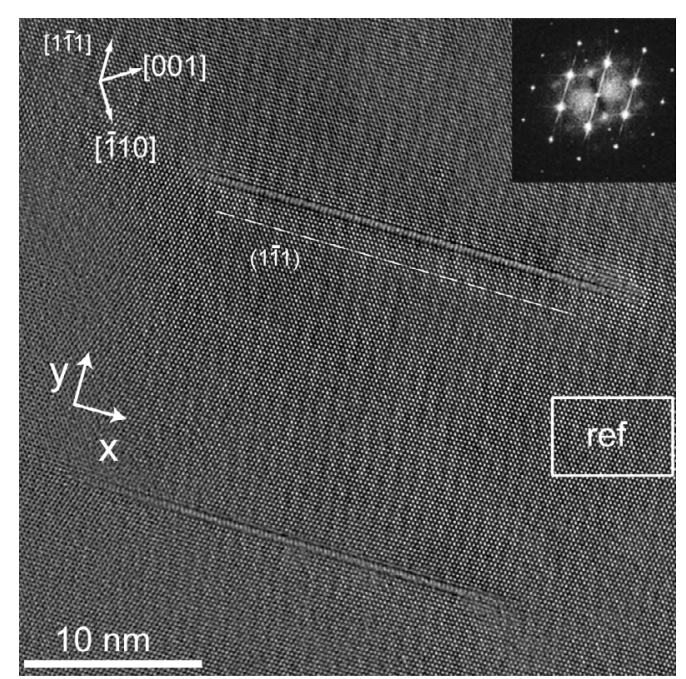

Fig. 2. Précipités dans A12198-T8. Image METHR, le faisceau d'électrons est aligné avec la direction [110] de la matrice d'aluminium.

Fig. 2. Precipitates in Al2198-T8. HREM image, the electron beam is aligned along the [110] direction of the aluminum matrix.

précipité crée un champ de déformation dans la matrice qui peut être directement mesuré en utilisant GPA à partir des images de METHR. Néanmoins, une bonne résolution est nécessaire à cause du faible ordre de grandeur des champs de déplacement mesurés, d'où l'utilisation d'un microscope équipé d'un correcteur d'aberration sphérique. Notons toutefois qu'avec cette technique, aucune information sur la composante du champ de déplacement parallèle au faisceau d'électrons ne peut être facilement obtenue puisqu'on image la projection de l'échantillon perpendiculairement au faisceau d'électrons. Enfin, pour mesurer les déformations dans la matrice en utilisant GPA, une zone considérée comme exempte de déformation et qui sert de référence $(\varepsilon=0)$ doit être définie (rectangle sur la Fig. 2).

La figure 3 montre les cartes des déformations $\varepsilon_{x x}, \varepsilon_{y y}$ et $\varepsilon_{x y}$ mesurées dans le plan (110) dans la matrice au voisinage du précipité. L'axe $x$ est choisi parallèlement à la trace du plan (11̄1) de la matrice d'aluminium, soit la direction [1112].

Le champ de déformation $\varepsilon_{x x}$ est présenté sur la figure 3 a. Les mesures montrent une compression $\left(\varepsilon_{x x} \leq-5 \%\right.$, en noir) et une dilatation ( $\varepsilon_{x x} \geq+5 \%$, en blanc) de la matrice aux deux extrémités du précipité. Les zones de compression et de dilatation s'échangent à l'autre extrémité d'un même précipité. Au voisinage du centre du précipité, la matrice n'est pas déformée dans la direction $x$.

La figure $3 \mathrm{~b}$ montre la composante $\varepsilon_{y y}$. Les positions par rapport au précipité, de la compression et de la dilatation maximale sont échangées en comparaison de celles observées sur $\varepsilon_{x x}$.

Enfin $\varepsilon_{x y}$ (Fig. 3c) montre une dilatation de la matrice aux extrémités du précipité, et une compression en son centre.

\section{Modélisations des champs de déformation}

L'observation directe montre que les plans atomiques de type $\{111\}$ de la matrice qui traversent le précipité sont décalés à la traversée du précipité (Fig. 4). L'image METHR (Fig. 4a) est déformée suivant la direction verticale en figure $4 \mathrm{~b}$ afin de mettre en évidence plus facilement le désalignement des plans atomiques à la traversée du précipité. En l'absence du précipité, les rangées atomiques de la matrice sont parfaitement alignées.

Ce décalage, facilement mesurable directement sur l'image HREM, correspond à un tiers de la distance entre deux plans (111), soit exactement le module du vecteur de faute correspondant à une faute d'empilement de type $a / 6\langle 112\rangle$. Cette faute d'empilement n'existant pas en dehors du précipité, car aucun décalage de plans n'est observable dans la matrice hors du précipité, est donc circonscrite au précipité. Cette faute est forcément bordée par des dislocations qui se trouvent être des dislocations partielles de la structure cubique à faces centrées de la matrice. Ces dislocations partielles sont modélisées à partir d'une dislocation parfaite de l'aluminium de vecteur de Burgers $a / 2[110]$ dissociée en deux dislocations partielles de vecteurs de Burgers selon :

$$
a / 2[110] \rightarrow a / 6[21 \overline{1}]+a / 6[121]
$$

Seule la composante du vecteur de Burgers perpendiculaire à la direction des électrons, ici [110] est observable en MET (Fig. 5). La composante du vecteur de Burgers perpendiculaire à [110] pour la dislocation 

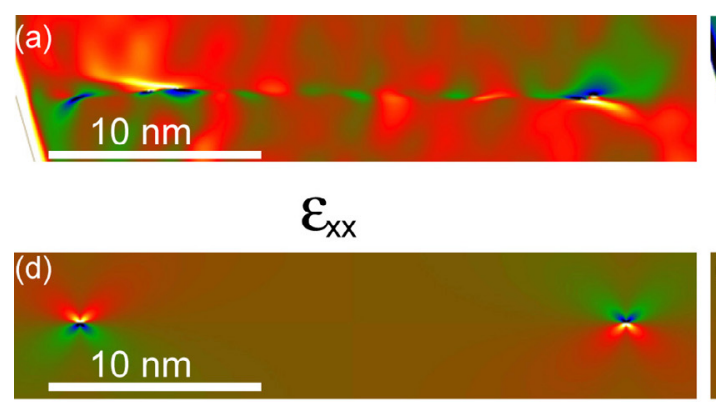

$\varepsilon_{\mathrm{xx}}$

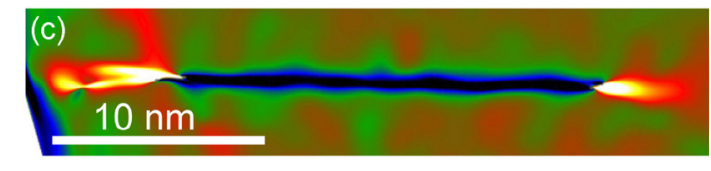

$\varepsilon_{\mathrm{xy}}$

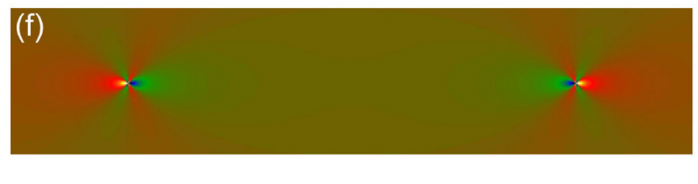

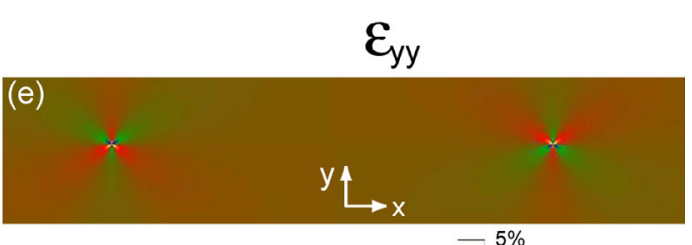

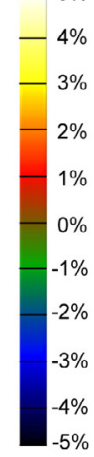

Fig. 3. Cartes des déformations mesurées pour les composantes $\varepsilon_{x x}(\mathrm{a}), \varepsilon_{y y}$ (b) et $\varepsilon_{x y}$ (c) et modélisées pour les composantes $\varepsilon_{x x}$ (d), $\varepsilon_{y y}$ (e) et $\varepsilon_{x y}$ (f).

Fig. 3. Experimental strains $\varepsilon_{x x}(a), \varepsilon_{y y}(b)$ and $\varepsilon_{x y}(c)$ and corresponding modeled strains $\varepsilon_{x x}(d), \varepsilon_{y y}(e)$ and $\varepsilon_{x y}(f)$.
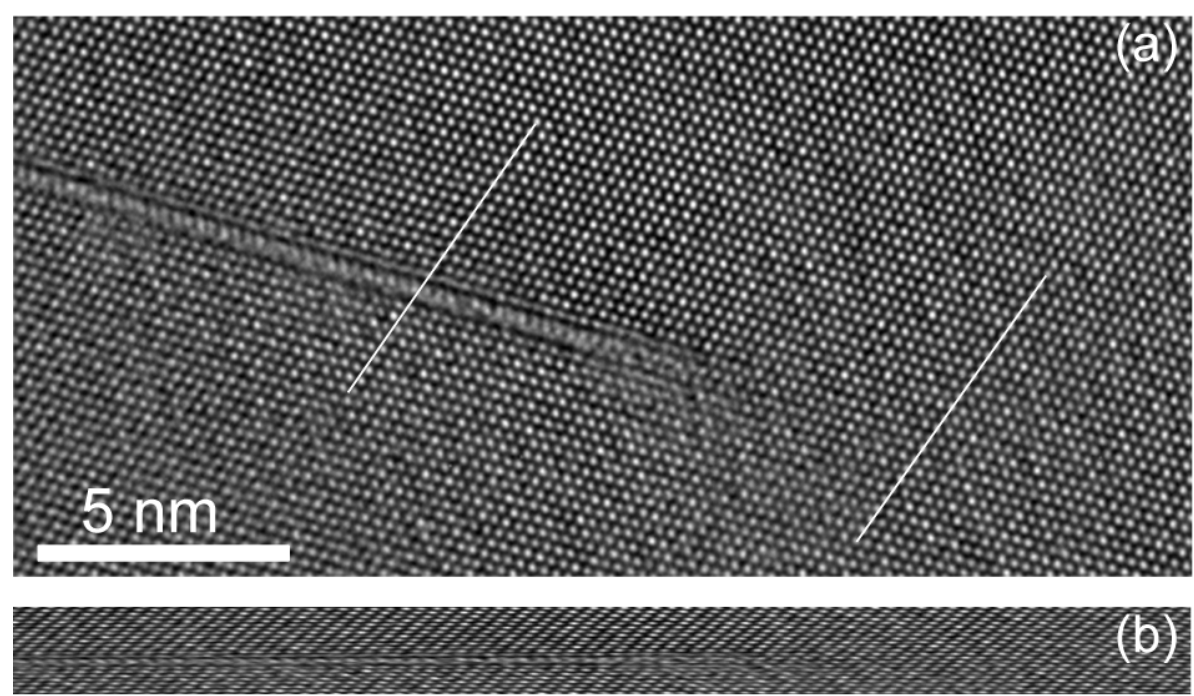

Fig. 4. Mise en évidence du décalage des plans atomiques à la traversée du précipité dans Al2198T8 : (a) image METHR, le faisceau d'électrons est aligné avec la direction [110] de la matrice d'aluminium; (b) une partie de la même image déformée dans la direction verticale pour mettre en évidence le non alignement des plans atomiques à la traversée du précipité.

Fig. 4. The atomic planes are shifted when the precipitate is crossed in Al2198-T8. (a) HRTEM image, the electron beam is aligned along the [110] direction of the aluminum matrix; (b) part of the same image but shrunk in the vertical direction to show the shifted atomic planes when the precipitate is crossed. 


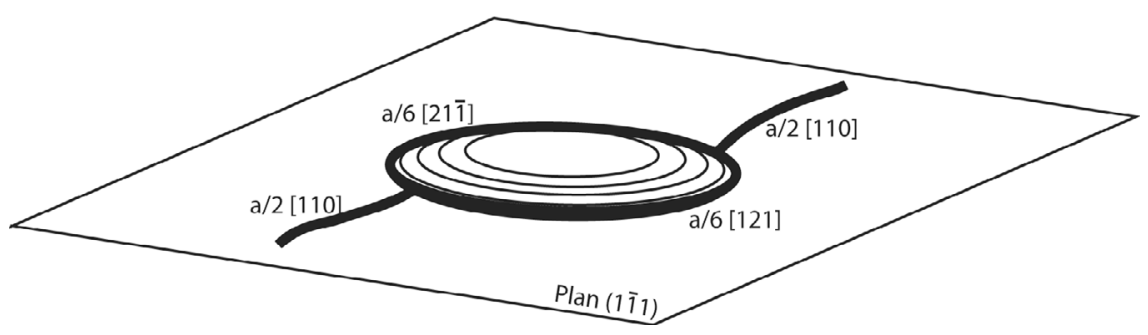

(a)

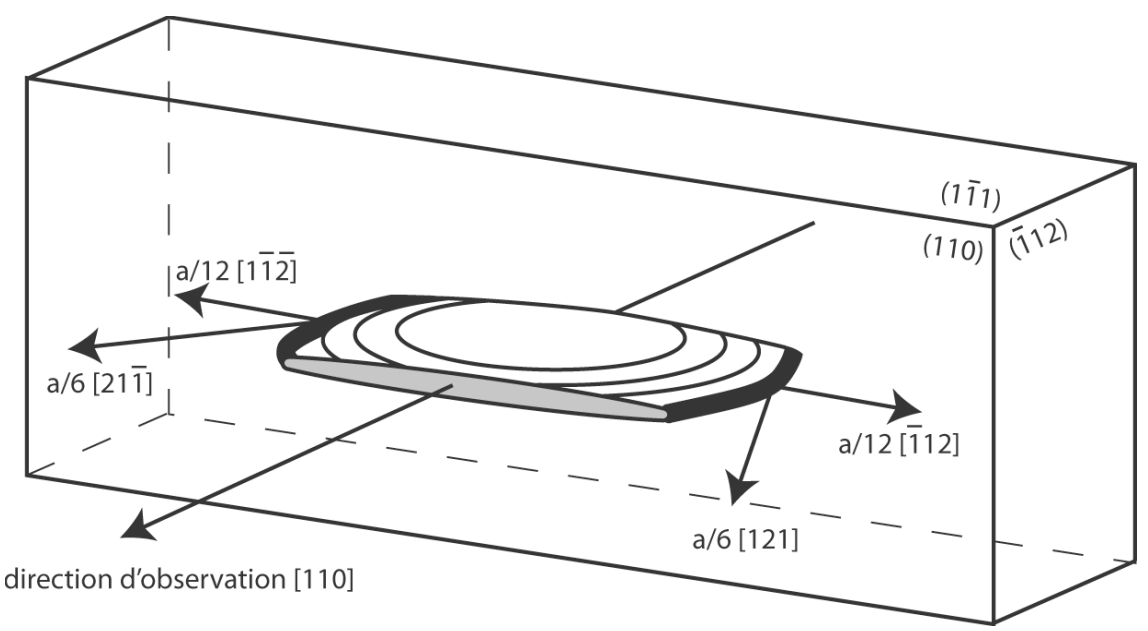

(b)

Fig. 5. (a) Vue schématique d'un précipité lenticulaire et de la dislocation dissociée utilisée pour le modéliser (b) Vue schématique de la lame mince de MET montrant les vecteurs de Burgers des dislocations entourant le précipités et la composante perpendiculaire.

Fig. 5. (a) Schematic view of a disc-shaped precipitate and of the dislocations used to modeled it; (b) schematic view of the TEM thin the foil showing the Burgers vectors of the partial dislocations bordering the precipitate and the component of these Burgers vectors perpendicular to the beam that contribute to the measurable strain.

$\vec{b}=a / 6[21 \overline{1}]$ est $a / 12[1 \overline{1} \overline{2}]$. De la même manière, la composante du vecteur de Burgers perpendiculaire à [110] pour la dislocation $\vec{b}=a / 6[121]$ est $a / 12[\overline{1} 12]$. Le champ de déformation autour du précipité est modélisé, en première approximation, comme la somme des champs de déformation d'un dipôle de dislocations [7] à partir de :

$\varepsilon_{x x}=\frac{-b}{4 \pi(1-v)} X_{2}\left(\frac{(3-2 v) X_{1}^{2}+(1-2 v) X_{2}^{2}}{\left(X_{1}^{2}+X_{2}^{2}\right)^{2}}\right)$

$\varepsilon_{y y}=\frac{b}{4 \pi(1-v)} X_{2}\left(\frac{(1-2 v) X_{1}^{2}+(2 v-1) X_{2}^{2}}{\left(X_{1}^{2}+X_{2}^{2}\right)^{2}}\right)$

$\varepsilon_{x y}=\frac{b}{4 \pi(1-v)} X_{1}\left(\frac{X_{2}^{2}-X_{1}^{2}}{\left(X_{1}^{2}+X_{2}^{2}\right)^{2}}\right)$ qui donnent, en élasticité isotrope, les expressions du champ de déformations à la position relative $\left(X_{1}, X_{2}\right)$ au cœur de la dislocation de vecteur de Burgers $b(=a \sqrt{6} / 12)$, avec $v(=0,347)$ le coefficient de Poisson du matériau.

Le résultat de cette modélisation est présenté en figures $3 d$, e et $f$.

Pour $\varepsilon_{x x}$ les déformations de la matrice à proximité des extrémités et du centre du précipité sont en bon accord avec l'expérience. En particulier, l'échange entre compression et dilatation est retrouvé à l'autre extrémité de précipité. Pour $\varepsilon_{y y}$ et $\varepsilon_{x y}$, les déformations de la matrice aux extrémités du précipité sont également en bon accord, mais de larges différences sont observées lorsqu'on se rapproche du centre du précipité. Bien évidemment, une modélisation basée seulement sur une boucle planaire de 
dislocations ne peut pas rendre compte des déformations $\varepsilon_{y y}$ et $\varepsilon_{x y}$ au centre du précipité, car même si l'épaisseur des précipités est faible, elle n'est pas négligeable. Une modélisation complète nécessitera donc d'inclure l'influence de cette épaisseur. Cependant un calcul élastique complet nécessiterait la connaissance des constantes élastiques de la phase $T_{1}$, qui ne sont pas disponibles pour l'instant.

Enfin, il est à noter que pour simuler les interactions des dislocations avec les précipités et pouvoir ainsi apporter de nouveaux éléments de compréhension sur le durcissement structural des alliages d'aluminium, il est nécessaire de connaître au voisinage des précipités les champs de contraintes dans la matrice. Ceux-ci peuvent être simplement déduits des champs de déformation par application de la loi de Hooke.

\section{Conclusions}

Il a été montré qu'il est possible de mesurer des déformations autour d'un précipité lenticulaire inclus dans une matrice cristalline. La structure complexe du précipité ne permet pas de connaître ses constantes élastiques, donc d'estimer a priori son interaction avec la matrice. Cependant, les déformations mesurées sont en partie décrites par un modèle simple basé sur la théorie élastique des dislocations. L'influence de l'épaisseur du précipité devra néanmoins être prise en compte par le modèle.

\section{Références}

[1] R. Rioja, Mater. Sci. Eng. A257 (1998) 100-107

[2] P. Donnadieu, Y. Shao, F. De Geuser, Acta Mater. 59 (2011) 462-472

[3] J.M. Howe, J. Lee, Metallurgical Transactions A 19A (1988) 2911-2920

[4] M. Hÿtch, E. Snoeck, R. Kilaas, Ultramicroscopy 74 (1998) 131-146

[5] J. Douin, P. Donnadieu, F. Houdellier, Acta Mater. 58 (2010) 5782-5788

[6] W. Tirry, D. Schryvers, Nature Materials 8 (2009) 752-757

[7] J.P. Hirth, J. Lothe, Theory of dislocations, John Wiley and Son, New York, 1982 